\title{
Cognitive Impairment and Related Causes in Hemodialysis Patients
}

\author{
Elham Shahraki (iD) ${ }^{1,}$, Mansoor Shakiba ${ }^{2}$, Melika Gholampour Yazdi ${ }^{1}$ and Narjes Sargolzaie (iD ${ }^{3}$ \\ ${ }^{1}$ Department of Internal Medicine, Ali Ibne Abitaleb Hospital, Zahedan University of Medical Sciences, Zahedan, Iran \\ ${ }^{2}$ Department of Psychiatry, Baharan Psychatric Hospital, School of Medicine, Zahedan University of Medical Sciences, Zahedan, Iran \\ ${ }^{3}$ Health Promotion Research Center, Zahedan University of Medical Sciences, Zahedan, Iran \\ "Corresponding author: Department of Internal Medicine, Ali Ibne Abitaleb Hospital, Zahedan University of Medical Sciences, Zahedan, Iran. Email: shahrekie@gmail.com
}

Received 2021 May 03; Revised 2021 December 07; Accepted 2022 January 15.

\begin{abstract}
Background: End-stage renal disease (ESRD) is the stage of renal failure that requires renal replacement therapy such as hemodialysis. Despite the beneficial effects of hemodialysis on patient survival and progress in the treatment of various disorders in these patients, patients still suffer from numerous complications, such as neurological and cognitive disorders, which do not improve with dialysis.

Objectives: This study aimed to evaluate cognitive impairment in hemodialysis patients in Zahedan, Iran, and design a model to predict the risk.

Methods: This cross-sectional study was performed on 114 hemodialysis patients in Zahedan. Due to the socioeconomic status of the people of this region and the lack of a similar study in this part of Iran, the mini-mental state examination (MMSE) was performed. An information checklist, including age, gender, lifetime frequency of hemodialysis, and duration of hemodialysis, was completed by the investigator.

Results: The MMSE score showed that cognitive impairment was common in ESRD patients, and its prevalence was significantly higher in elderly patients. Patients' cognitive function was significantly related to the lifetime frequency and duration of dialysis. Based on these results, we designed a model to predict the likelihood of cognitive impairment.

Conclusions: The results show that cognitive impairment is a common problem among ESRD patients. Dementia is associated with multiple risks such as death, withdrawal from dialysis, hospitalization, and exacerbation of disability in ESRD patients. Therefore, effective recognition and management of these disorders can improve clinical care.
\end{abstract}

Keywords: End-Stage Renal Disease, ESRD, Hemodialysis, Cognitive Function, Dementia

\section{Background}

Renal dysfunction gradually leads to end-stage renal disease (ESRD), the stage of renal failure that requires renal replacement therapy (1). In recent years, the incidence and prevalence rates of ESRD have increased in Iran and globally following a surge in the prevalence of diabetes and hypertension (2). The number of hemodialysis patients has doubled in recent decades, and these patients face a variety of problems (3), such as physical, psychological, and social problems, including cognitive impairment directly related to the fact that a further decline in renal function is associated with the exacerbation of cognitive disorders (4).

In some studies, the prevalence of cognitive impairment in hemodialysis patients was reported to be $30-60 \%$, which is about twice that of non-hemodialysis patients (5). In other studies, the prevalence of cognitive impairment was reported to be $50-78 \%$, which was higher in ESRD patients than in controls (6). This increase occurs due to var- ious reasons, including uremia, inflammation, cardiovascular disease, stroke, and various metabolic disorders (6, 7). Kidney and brain functions are interrelated, and both organs are affected by the cardiovascular system and blood pressure, and any changes and disorders in kidney function affect brain function as well $(8,9)$. Kidney dysfunction can be associated with brain dysfunction in various forms, one of which is cognitive disorders (10). Cognitive disorders in dialysis patients are associated with decreased patient cooperation, continuation of dialysis, treatment costs, mortality, and hospitalization of these patients (6). The epidemiological study is the main step to evaluate the clinical impact of cognitive impairment and planning management strategies.

\section{Objectives}

Given that no study has been conducted in Zahedan, we sought to assess the prevalence and causes of cogni- 
tive impairment in hemodialysis patients in Zahedan, Iran, and design a model to predict the amount of cognitive impairment risk.

\section{Methods}

In this descriptive cross-sectional study, all hemodialysis patients aged less than 60 years old with at least 6 months of hemodialysis at Ali Ibn Abitaleb and Khatam AlAnbia hospitals were enrolled from April to October 2019 in Zahedan, Iran. The inclusion criteria included patients with no history of known mental illness, psychosis, depression, mental retardation, or stroke. All patients have hemoglobin $(\mathrm{Hb})>10 \mathrm{mg} / \mathrm{dL}$ and $\mathrm{Kt} / \mathrm{V}>1.2(\mathrm{~K}$, dialyzer clearance of urea; $\mathrm{t}$, dialysis time; $\mathrm{V}$, volume of distribution of urea).

Exclusion criteria included patients with a history of transplant rejection, recent infection (past 3 months) and admission, uncontrolled hypertension, uncontrolled diabetes mellitus, or unwillingness to participate. Finally, 114 patients were enrolled in this study according to the inclusion and exclusion criteria. The Ethics Committee of Zahedan University of Medical Sciences approved this study (code: IR.ZAUMS.REC.1397.62). After obtaining patients' consent, an information checklist, including age, gender, lifetime frequency of hemodialysis, and duration of hemodialysis, was completed by the researcher during interviews with patients. Patients underwent a thorough examination, as well as a neurological examination, by the researcher.

Patients' cognitive function was assessed by the minimental state examination (MMSE). Based on the scores obtained on this scale, patients were assigned to one of 3 groups: normal (score 24 and above), suspected of cognitive impairment (score 18 - 23), and cognitive problem (score less than 18). The mini-mental state examination evaluates orientation, registration, calculation, recent memory, visuospatial thinking, and language situation. The reliability and validity of this questionnaire in Iran were proven by Seyedian et al. (Cronbach $\alpha=0.81$ ) (11). The data were analyzed using SPSS version 20 (SPSS Inc, Chicago, Ill, USA).

Chi-square and analysis of variance (ANOVA) were used to analyze the data. The multivariate linear regression test with backward elimination technique was used for the final model predicting the MMSE score. A P-value of less than 0.05 was considered significant.

\section{Results}

As mentioned above, 114 hemodialysis patients were enrolled in the present study, $48 \%$ of whom were male, and $52 \%$ of whom were female. Also, $68 \%$ had normal cognitive impairment, $25 \%$ had suspected cognitive impairment, and $7 \%$ had impaired cognitive impairment. The mean age for patients without cognitive impairment was $42 \pm 8$ years, for suspected patients was $50.7 \pm 10$ years old, and for patients with problems was $49 \pm 10$ years old. The results showed a significant relationship between age, lifetime frequency of dialysis, and duration of dialysis (Table 1). There was no significant difference between the 2 sexes in cognitive impairment.

Table 1. The Severity of Cognitive Impairment in Hemodialysis Patients by Age, Frequency of Dialysis, Duration of Dialysis, and Gender

\begin{tabular}{|ccccc}
\hline \multirow{3}{*}{ Characteristic Severity By MMSE } & \multirow{2}{*}{ P-Value } \\
\cline { 2 - 4 } & Normal & Suspicious & $\begin{array}{c}\text { Cognitive } \\
\text { Problem }\end{array}$ & \\
\hline Age (y) & & & & \\
\hline $20-$ & $28(68)$ & $4(25)$ & $1(5)$ & 0.000 \\
40 & $49(66)$ & $25(25)$ & $7(8)$ & \\
\hline $\begin{array}{c}40- \\
60\end{array}$ & 31 & 49 & 73 & 0.003 \\
\hline $\begin{array}{l}\text { Lifetime } \\
\text { frequency } \\
\text { of dialysis }\end{array}$ & 442 & 567 & 630 & 0.000 \\
\hline $\begin{array}{l}\text { Duration of } \\
\text { dialysis (d) }\end{array}$ & & $14(25)$ & $3(5)$ & \\
\hline \begin{tabular}{l} 
Gender \\
\hline Male
\end{tabular} & $38(69)$ & & & \\
\hline $\begin{array}{l}\text { Fe- } \\
\text { male }\end{array}$ & $39(66)$ & $15(25)$ & $5(8)$ & \\
\hline
\end{tabular}

${ }^{\mathrm{a}}$ Values are expressed as No. (\%).

Considering that cognitive disorders had a significant relationship with age and duration, and frequency of dialysis. According to the obtained results, we found this model to predict the MMSE score, which can be predicted by these items according to the formula obtained (MMSE score $=$ 35.33 - $[0.429 \times$ age $]-[0.336 \times$ dialysis duration $]-[0.239 \times$ dialysis time]).

Based on the multivariate linear regression test by the backward method, the final model predicting the MMSE score is summarized in the table 2. As can be seen, this model predicts $0.47 \%$ of the MMSE variance, and the prediction coefficient of each variable in the regression model (beta) is summarized in Table 2.

\section{Discussion}

The results of this study showed that cognitive disorders were common in hemodialysis patients, and about one-third of these patients had cognitive impairment, which was clearly higher than the rate in the general population (3.2\%) (12). The results of our study are in line with 


\begin{tabular}{lccccc}
\hline Table 2. Results of Multivariate Regression Analysis to Predict the MMSE Score \\
\hline Parameter & B & SE & Beta & $\boldsymbol{t}$ & P-Value \\
\hline Constant & 35.33 & 1.26 & - & 27.89 & 0.000 \\
Age & -0.148 & 0.024 & -0.429 & -6.19 & 0.000 \\
Dialysis duration & -0.052 & 0.011 & -0.366 & -4.66 & 0.000 \\
Lifetime frequency & -0.005 & 0.002 & -0.239 & -3.02 & 0.003 \\
\hline
\end{tabular}

the results of other studies, such as in the United States that the population of hemodialysis patients is 700,000 , two-thirds of whom have cognitive impairment (13). Other studies have also reported a high prevalence of cognitive impairment in ESRD patients. Eslami Amirabadi et al. in a study performed in Tehran reported this rate to be $47 \%$ (9). Kalirao et al. found that two-thirds of hemodialysis and peritoneal dialysis patients had cognitive impairments, which is consistent with our finding if we consider the total number of suspected and problematic cases (7); also, it is consistent with the results of Tamadon et al., who showed that $28.2 \%$ of ESRD patients had severe cognitive impairment, which was significantly higher than the rate of cognitive impairment in patients with chronic kidney disease (14). Murray et al. reported that more than one-third of hemodialysis patients had severe cognitive impairment (15); this high prevalence can have several causes. For instance, kidney problems can affect brain function. Aging, diabetes mellitus, hypertension, hypercholesterolemia (along with chronic inflammation), oxidative stress, and hypercoagulopathy can also cause vascular and endothelial damage, affecting the kidneys and the brain. Uremic toxins have a direct effect on neurons through the neurodegenerative mechanism (10).

Further, studies have shown that dialysis patients have brain atrophy, reduced hippocampal volume, thinning of the cerebral cortex in the temporomandibular joint and white matter damage in the fornix, inner capsule, and thalamus, all of which are similar to aging changes. This evidence can be seen on imaging even before changes the hemodialysis patient's functional and cognitive status (16). On the other hand, chronic inflammation in hemodialysis patients increases the level of cytokines, impairs the secretion of neurotransmitters, and exacerbates neurological and cognitive disorders (17). Studies have also demonstrated that decreased cerebral blood flow during dialysis and continued dialysis during the month are associated with cerebrovascular disease and decreased cerebral function in these patients (18). In general, the common mechanisms of increased cognitive impairment in hemodialysis patients increase by vascular disease, oxidative stress, silent or overt cerebral infarction, hyperhomocysteinemia, impaired renal function, anemia, inadequate dialysis, malnutrition, and chronic inflammation (10).

Our study indicated that the increased frequency of dialysis and the number of years of dialysis were associated with a higher rate of cognitive impairment. The results were consistent with the results obtained by Sarnak et al., as well as the hypothesis that an increase in the speed of atherosclerosis is associated with a higher MMSE score (19). Studies have shown that brain function improves following kidney transplantation (18), and efforts should be made to provide kidney transplantation for these patients more quickly. The rate of cognitive disorders in hemodialysis patients is high due to the increased prevalence of diabetes and hypertension, followed by renal dysfunction and increased need for dialysis, and these patients need special care. Cognitive impairment is associated with increased mortality and hospitalization (13). According to our model, elderly patients who have been dialyzed longer and patients who have been on dialysis more often are at a higher risk for cognitive impairment. Other studies confirm our results (19). It is recommended that during periodic visits of hemodialysis patients, these patients should be examined for cognitive impairment and, if necessary, treatment should be initiated as soon as possible, and, if possible, kidney transplantation should be performed more quickly.

Moreover, the small number of samples and the lack of imaging, along with not examining the inflammatory factors, were the limitations of our study.

\section{Footnotes}

Authors' Contribution: Study concept and design: E. Sh., and M.Sh.; Analysis and interpretation of data: N. S., and M. Gh.; Drafting of the manuscript: E. Sh.; Critical revision of the manuscript for important intellectual content: E. Sh., M. Gh., and M. Sh.; Statistical analysis: N. S..

Conflict of Interests: The authors declare no conflicts of interest.

Ethical Approval: The Ethics Committee of Zahedan University of Medical Sciences approved this study (code: IR.ZAUMS.REC.1397.62).

Funding/Support: The authors declare no funding/support.

Informed Consent: Written informed consent was obtained from all participants.

\section{References}

1. Raeesi F, Nasehi AA, Ekhtiari M. Frequency of Depression in Hemodialysis Patients in Imam Khomeini Hospital. Adv Cogn Psychol. 2005;7(2):55-9. eng. 
2. Mousavi SS, Soleimani A, Mousavi MB. Epidemiology of end-stage renal disease in Iran: a review article. Saudi J Kidney Dis Transpl. 2014;25(3):697-702. doi: 10.4103/1319-2442.132242. [PubMed: 24821181].

3. Mahmoodi S, Salehnejad G. The Comparison of Depression in Hemodialysis and Renal Transplantation Patients. Life Sci J. 2012;9(4):5754-8.

4. Renczes E, Maronek M, Gaal Kovalcikova A, Vavrincova-Yaghi D, Tothova L, Hodosy J. Behavioral Changes During Development of Chronic Kidney Disease in Rats. Front Med (Lausanne). 2019;6:311. doi: 10.3389/fmed.2019.00311. [PubMed: 31998731]. [PubMed Central: PMC6962109].

5. Bugnicourt JM, Godefroy O, Chillon JM, Choukroun G, Massy ZA. Cognitive disorders and dementia in CKD: The neglected kidney-brain axis. J Am Soc Nephrol. 2013;24(3):353-63. doi: 10.1681/ASN.2012050536. [PubMed: 23291474].

6. Gupta A, Mahnken JD, Johnson DK, Thomas TS, Subramaniam D, Polshak T, et al. Prevalence and correlates of cognitive impairment in kidney transplant recipients. BMC Nephrol. 2017;18(1):158. doi: 10.1186/s12882-017-0570-1. [PubMed: 28499360]. [PubMed Central: PMC5429555].

7. Kalirao P, Pederson S, Foley RN, Kolste A, Tupper D, Zaun D, et al. Cognitive impairment in peritoneal dialysis patients. Am J Kidney Dis. 2011;57(4):612-20. doi: 10.1053/j.ajkd.2010.11.026. [PubMed: 21295896]. [PubMed Central: PMC3121243].

8. Davey A, Elias MF, Robbins MA, Seliger SL, Dore GA. Decline in renal functioning is associated with longitudinal decline in global cognitive functioning, abstract reasoning and verbal memory. Nephrol Dial Transplant. 2013;28(7):1810-9. doi: 10.1093/ndt/gfs470. [PubMed: 23166308]. [PubMed Central: PMC3707524].

9. Eslami Amirabadi M, Hosein DK, Nasrollahi A, Norouzian M, Bozorg B, Amjadi Kivi SM, et al. Cognitive dysfunction in hemodialysis patients and its related factors. Res Med. 2014;38(1):53-9. eng.

10. Afsar B, Sag AA, Yalcin CE, Kaya E, Siriopol D, Goldsmith D, et al. Brainkidney cross-talk: Definition and emerging evidence. EurJIntern Med. 2016;36:7-12. doi: 10.1016/j.ejim.2016.07.032. [PubMed: 27531628].

11. Seyedian M, Falah M, Nourouzian M, Nejat S, Delavar A, Ghasemzadeh
HA. Validity of the Farsi Version of Mini-Mental State Examination. $J$ Iran Med Counc. 2008;25(4):408-14.

12. Navipour E, Neamatshah M, Barabadi Z, Neamatshah M, Keykhosravi A. Epidemiology and Risk Factors of Alzheimer's disease in Iran: A Systematic Review. Iran J Public Health. 2020. doi: 10.18502/ijph.v48i12.3544.

13. Wolfgram DF. Intradialytic Cerebral Hypoperfusion as Mechanism for Cognitive Impairment in Patients on Hemodialysis. J Am Soc Nephrol. 2019;30(11):2052-8. doi: 10.1681/ASN.2019050461. [PubMed: 31511363]. [PubMed Central: PMC6830804].

14. Tamadon MR, Adibimehr AR, Ghorbani R. Cognitive Impairment in Dialysis and Non-Dialysis Patients Suffering from Chronic Renal Failure and Comparing Them with a Control Group. Middle East J Rehabil Health. 2017;4(4). doi: 10.5812/mejrh.12815.

15. Murray AM, Tupper DE, Knopman DS, Gilbertson DT, Pederson SL, Li S, et al. Cognitive impairment in hemodialysis patients is common. Neurology. 2006;67(2):216-23. doi: 10.1212/01.wnl.0000225182.15532.40. [PubMed:16864811].

16. Chiu YL, Tsai HH, Lai YJ, Tseng HY, Wu YW, Peng YS, et al. Cognitive impairment in patients with end-stage renal disease: Accelerated brain aging? J Formos Med Assoc. 2019;118(5):867-75. doi: 10.1016/j.jfma.2019.01.011. [PubMed: 30744935].

17. Schiepers OJ, Wichers MC, Maes M. Cytokines and major depression. Prog Neuropsychopharmacol Biol Psychiatry. 2005;29(2):201-17. doi: 10.1016/j.pnpbp.2004.11.003. [PubMed: 15694227].

18. Findlay MD, Dawson J, Dickie DA, Forbes KP, McGlynn D, Quinn T, et al. Investigating the Relationship between Cerebral Blood Flow and Cognitive Function in Hemodialysis Patients. J Am Soc Nephrol. 2019;30(1):147-58. doi: 10.1681/ASN.2018050462. [PubMed: 30530658]. [PubMed Central: PMC6317612].

19. Sarnak MJ, Tighiouart H, Scott TM, Lou KV, Sorensen EP, Giang LM, et al. Frequency of and risk factors for poor cognitive performance in hemodialysis patients. Neurology. 2013;80(5):471-80. doi: 10.1212/WNL.ob013e31827fof7f. [PubMed: 23303848]. [PubMed Central: PMC3590049]. 